\title{
Review Article \\ Cardiovascular Changes in Animal Models of Metabolic Syndrome
}

\author{
Alexandre M. Lehnen, ${ }^{1,2}$ Bruno Rodrigues, ${ }^{3}$ Maria Cláudia Irigoyen, ${ }^{4}$ \\ Kátia De Angelis, ${ }^{5}$ and Beatriz D'Agord Schaan ${ }^{2}$ \\ ${ }^{1}$ Laboratório de Experimentação Animal e Laboratório de Cardiologia Celular e Molecular, \\ Instituto de Cardiologia do Rio Grande do Sul/Fundação Universitária de Cardiologia do Rio Grande do Sul, Porto Alegre, Brazil \\ ${ }^{2}$ Divisão de Endocrinologia, Hospital de Clínicas de Porto Alegre, Universidade Federal do Rio Grande do Sul, Porto Alegre, Brazil \\ ${ }^{3}$ Laboratório do Movimento Humano, Universidade São Judas Tadeu, São Paulo, Brazil \\ ${ }^{4}$ Unidade de Hipertensão, Instituto do Coração (InCor), Faculdade de Medicina da Universidade de São Paulo, São Paulo, Brazil \\ ${ }^{5}$ Laboratório de Fisiologia Translacional, Universidade Nove de Julho, São Paulo, Brazil
}

Correspondence should be addressed to Beatriz D’Agord Schaan; beatrizschaan@gmail.com

Received 12 December 2012; Revised 6 February 2013; Accepted 12 February 2013

Academic Editor: Tomohiko Sasase

Copyright ( 2013 Alexandre M. Lehnen et al. This is an open access article distributed under the Creative Commons Attribution License, which permits unrestricted use, distribution, and reproduction in any medium, provided the original work is properly cited.

\begin{abstract}
Metabolic syndrome has been defined as a group of risk factors that directly contribute to the development of cardiovascular disease and/or type 2 diabetes. Insulin resistance seems to have a fundamental role in the genesis of this syndrome. Over the past years to the present day, basic and translational research has used small animal models to explore the pathophysiology of metabolic syndrome and to develop novel therapies that might slow the progression of this prevalent condition. In this paper we discuss the animal models used for the study of metabolic syndrome, with particular focus on cardiovascular changes, since they are the main cause of death associated with the condition in humans.
\end{abstract}

\section{Introduction}

According to the International Diabetes Federation [1], metabolic syndrome (MS) is clinically characterized by central obesity (waist circumference $\geq 94 \mathrm{~cm}$ for men and $\geq 80 \mathrm{~cm}$ for women), and at least two of these risk factors: high triglyceride levels $(\geq 150 \mathrm{mg} / \mathrm{dL})$; low HDL cholesterol $(\leq 40 \mathrm{mg} / \mathrm{dL})$; high blood pressure levels (systolic blood pressure $\geq 130 \mathrm{mmHg}$ and/or diastolic blood pressure $\geq 85 \mathrm{mmHg}$ ); and fasting plasma glucose levels $\geq 100 \mathrm{mg} / \mathrm{dL}$. This cluster of cardiovascular risk factors is intrinsically related to increased incidence of diabetes mellitus [2] and cardiovascular mortality [3].

Studies have demonstrated several MS-induced abnormalities of cardiac geometry and function. Both increased left ventricular mass and relative wall thicknesses, as well as rapid deceleration time, have been found in hypertensive subjects with MS when compared with a hypertensive cohort without the syndrome [4]. On the other hand, patients with MS have presented left ventricular diastolic dysfunction independent of ventricular mass [5]. In addition, it is well known that insulin resistance plays a key role in MS and as such contributes to the development of premature cardiovascular atherosclerosis, independent of the association with diabetes and obesity [6-8]. It seems reasonable to assume that the association of several risk factors, as in the MS, favors an increased incidence of cardiovascular diseases and death risks in humans. The main cardiovascular complications observed in MS can be seen in Figure 1. Thus, a better understanding of the pathophysiological mechanisms of this syndrome becomes of paramount importance in clinical practice.

Studies on animal models can be relevant as they mimic the aspects of the human disease as the development and maintenance of MS characteristics, particularly obesity, type 2 diabetes, dyslipidemia, and hypertension. Experimental models of MS may be genetic, chemically induced, or diet induced. Thus, the aim of this paper is to both describe and discuss the animal models used for the study of MS. A special 


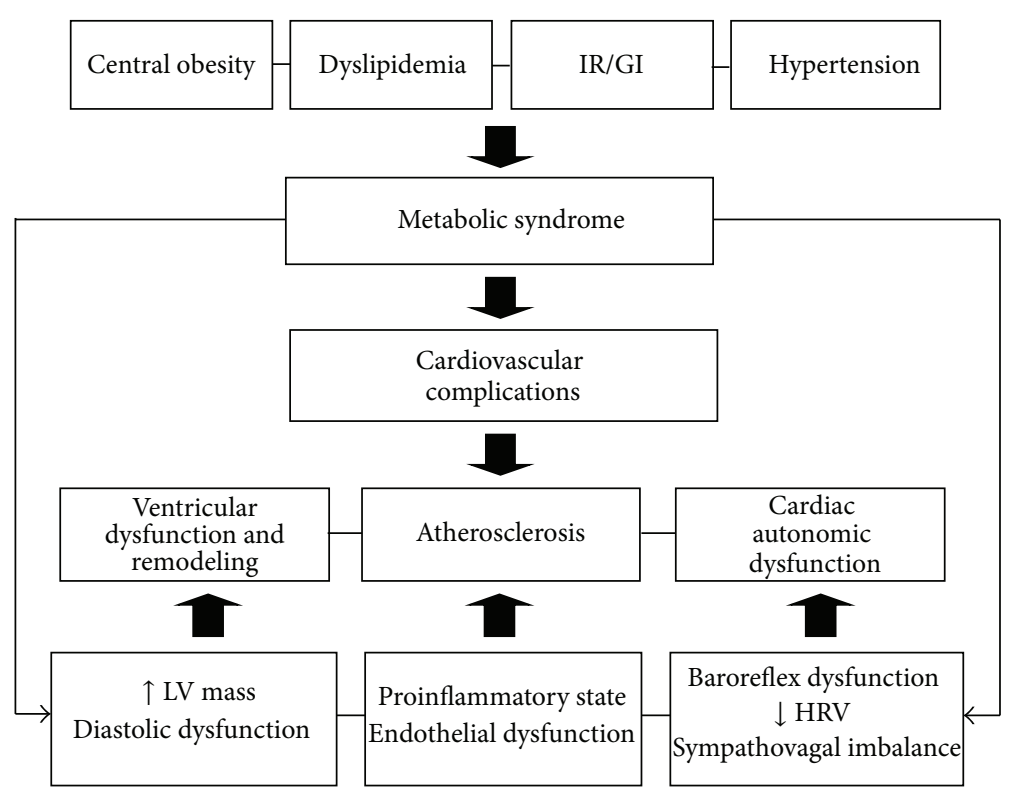

FIGURE 1: Components of the metabolic syndrome and its relation to the principal cardiovascular phenotypes. IR/GI: insulin resistance/glucose intolerance; HRV: heart rate variability; LV: left ventricular.

focus was given to cardiovascular changes which can be seen in Tables 1,2 , and 3 .

\subsection{Genetic Models}

1.1.1. $d b / d b$ Mouse. The $d b / d b$ mouse (BKS.Cgm+/+Leprdb/ j) is a genetic model widely used as MS animal model, as it presents a leptin receptor mutation which causes hyperglycemia and insulin resistance [9]. The cardiovascular changes observed in in vivo studies indicate an increased vascular contractility [10]. However, the evaluation of these animals with 8-9 weeks of age did not lead to changes in blood pressure as compared to their wild controls [11]. As the animals age (14-15 weeks), blood pressure rises, together with autonomic neural changes. Noradrenergic responsiveness of the heart is reduced and indications of sympathetic denervation are observed [12].

The increase in blood pressure of these animals is associated with an increase in plasma angiotensin converting enzyme activity and angiotensin II levels [11]. Using spectral methods for autonomic function evaluation, no changes in heart rate variability, blood pressure variability, or baroreflex function are observed $[11,13]$. In addition, $d b / d b$ mice show impaired cardiac functional reserve capacity during maximal beta-adrenergic stimulation (with dobutamine), which is associated with unfavorable changes in cardiac energy metabolism [14].

1.1.2. KKAy, ob/ob, and $d b / d b$ Mice. KKAy is a congenital strain established by the transduction of the yellow obese gene (Ay) into the moderate hyperglycemic KK strain [15]. KKAy mice are obese and have high blood pressure levels, increased urinary excretion of catecholamines, and exacerbated responses to sympathetic blockade, suggesting a sympathetic role in the genesis of their hypertension $[16,17]$.

The $o b / o b$ mice have a mutation in the ob gene resulting in leptin deficiency [18]. Short-term direct blood pressure measurements [19] suggested that the $o b / o b$ mice are hypotensive with low sympathetic nerve activity [20]. However, when blood pressure is measured chronically, that is, data are collected for $5 \mathrm{~s}$ every $2 \mathrm{~min}$ and are averaged for the light cycle (7 AM-4 PM) and the dark cycle (7 PM-5 AM) with radiotelemetry ( $24 \mathrm{~h}$ full-time), ob/ob mice remain hypertensive during the light period [21]. Interestingly, during the dark cycle, ob/ob mice show no difference in blood pressure. On examination of the data over $24 \mathrm{~h}$ (the dark and light cycles combined), $o b / o b$ mice are normotensive compared with control rats. With the use of radiotelemetry, blood pressure was measured $24 \mathrm{~h} /$ day, which obviously includes every activity performed by the animals. Thus, behavior and feeding may be especially relevant since the $o b / o b$ animals have leptin deficiency and eat throughout the day and at night, leading to the hypertension observed during the day. Furthermore, these animals develop left ventricular hypertrophy with decreased cardiac function at 24 weeks of age [22] and cardiac fibrosis after 20 weeks of age [23].

Finally, the $d b / d b(\mathrm{C} 57 \mathrm{BL} / \mathrm{Ks} J-\mathrm{db} / \mathrm{db})$ mice have inherited an autosomal recessive mutation in the leptin receptor gene present on chromosome 4 [9]. The metabolic alterations most frequently observed in this strain are hyperglycemia, hyperinsulinemia, hypertriglyceridemia, hypercholesterolemia, high levels of nonesterified fatty acids, and reduced HDL cholesterol [24]. In addition to these changes, 
TABLE 1: Main cardiovascular findings in genetic models of metabolic syndrome.

\begin{tabular}{|c|c|}
\hline Models & Cardiovascular changes \\
\hline \multirow{3}{*}{$d b / d b$ mouse } & (i) Increased vascular contractility \\
\hline & (ii) High blood pressure levels (14-15 weeks of age) \\
\hline & (iii) Sympathetic denervation (14-15 weeks of age) \\
\hline \multirow{2}{*}{ KKAy mice } & (i) High blood pressure levels \\
\hline & (ii) Sympathetic alterations \\
\hline \multirow{4}{*}{$o b / o b$ mice } & (i) Hypotensive with low sympathetic nerve activity \\
\hline & (ii) Cardiac fibrosis (20 weeks of age) \\
\hline & (iii) Left ventricular hypertrophy (24 weeks of age) \\
\hline & (iv) Decreased cardiac function (24 weeks of age) \\
\hline \multirow{4}{*}{$d b / d b$ mice } & (i) Vascular endothelial dysfunction \\
\hline & (ii) No or low blood pressure changes \\
\hline & (iii) No changes in heart rate variability \\
\hline & (iv) No changes in spontaneous baroreflex sensitivity \\
\hline \multirow{3}{*}{ Wistar Ottawa Karlsburg W } & (i) Impaired coronary function \\
\hline & (ii) Increased alpha(1)-adrenoceptor-mediated coronary constriction (3 and 10 months of age) \\
\hline & (iii) Seriously blunted beta-adrenoceptor-mediated coronary relaxation (16 months of age) \\
\hline \multirow{4}{*}{ Zucker obese rats } & (i) Diastolic dysfunction with preserved ejection fraction (9 weeks of age) \\
\hline & (ii) High blood pressure levels (12 weeks of age) \\
\hline & (iii) High resting sympathetic nerve activity \\
\hline & (iv) Reduced heart rate variability \\
\hline \multirow{3}{*}{ Zucker Diabetic Fatty } & (i) Increased myocardial fatty acid oxidation \\
\hline & (ii) Reduction of insulin-mediated myocardial glucose utilization (14 weeks of age) \\
\hline & (iii) Reduction of left ventricular chamber \\
\hline \multirow{3}{*}{ DahlS.Z-Lepr ${ }^{\mathrm{fa}} /$ Lepr $^{\mathrm{fa}}$} & (i) Diastolic dysfunction \\
\hline & (ii) Marked left ventricle hypertrophy and fibrosis \\
\hline & (iii) Myocardial oxidative stress \\
\hline \multirow{5}{*}{$\begin{array}{l}\text { Otsuka Long-Evans } \\
\text { Tokushima Fatty }\end{array}$} & (i) Diastolic dysfunction (15 weeks) \\
\hline & (ii) No changes in the blood pressure and heart rate \\
\hline & (iii) Extracellular fibrosis and abundant transforming growth factor- $\beta 1$ receptor II in the left ventricle \\
\hline & (iv) Low coronary flow reserve \\
\hline & (v) Increased coronary vascular resistance \\
\hline \multirow{4}{*}{ Goto-Kakizaki } & (i) Left ventricle remodeling with marked hypertrophy \\
\hline & (ii) Increased extracellular matrix deposition \\
\hline & (iii) Mild hypertension \\
\hline & (iv) Blunted vascular relaxation by acetylcholine and sodium nitroprusside \\
\hline
\end{tabular}

both infiltration with inflammatory cells and fibrosis were observed in the heart after 12 weeks of age. These mice also show vascular endothelial dysfunction, although no blood pressure changes are observed [25]. We studied the cardiovascular and autonomic phenotype of male $d b / d b$ mice and evaluated the role of angiotensin II AT(1) receptors. Radiotelemetry was used to monitor $24 \mathrm{~h}$ blood pressure in mice for 8 weeks. Although there were no changes in heart rate variability and spontaneous baroreflex sensitivity between control and $d b / d b$ mice, the results indicate an age-related increase in mean arterial pressure in $d b / d b$ mice, which can be reduced by the antagonism of angiotensin II AT(1) receptors [11].
1.1.3. Wistar Ottawa Karlsburg W(WOKW). Wistar Ottawa Karlsburg W (WOKW) rats were developed from a Wistar rat outbred strain of the BioBreeding Laboratories. As observed in humans, the features of MS in this model depend on polygenic factors and may be due to a single-gene mutation [26]. WOKW presents hyperphagia, which leads to obesity. In addition to obesity, this animal model shows other metabolic alterations, such as dyslipidemia, hyperinsulinemia, and impaired glucose tolerance. Specifically, insulin resistance in this animal model may be linked to a mutation on chromosome $3[26,27]$. Genomic scan studies have revealed a linkage of the MS and/or diabetes to a region on chromosome 3 (3q26-27), where the gene encoding adiponectin, apM1, is 
TABLE 2: Main cardiovascular findings in chemically induced animal models of metabolic syndrome.

\begin{tabular}{ll}
\hline Models & Cardiovascular changes \\
\hline MSG-induced & (i) High blood pressure levels \\
SHR & (iii) Deduced heart rate variability \\
& (iv) Increased cardiac sympathovagal balance \\
& (v) Increased systolic arterial pressure variability \\
\hline & (i) Systolic and diastolic dysfunction at rest \\
Streptozotocin & (ii) Reduced capacity for cardiac adjustment to \\
administration & volume overload \\
& (iii) Reduction in baroreflex-mediated \\
& bradycardia and tachycardia \\
& (iv) Impairment in cardiac vagal tone
\end{tabular}

MSG: monosodium glutamate; SHR: spontaneously hypertensive rat.

located [28]. One important clinical characteristic displayed by these animals was the impaired coronary function, due to increased alpha(1)-adrenoceptor-mediated coronary constriction (at 3 and 10 months of age), and to a seriously blunted beta-adrenoceptor-mediated coronary relaxation (at 16 months of age) [29].

1.1.4. Zucker Obese Rats (fa/fa). Zucker obese (ZO) rat model develops MS characterized by obesity since they are polyphagic due to a mutation in the leptin receptor [30,31], insulin resistance, hypertriglyceridemia, and hypertension [32]. Obesity leads to an inflammatory state which is linked to reduced insulin sensitivity and expression of GLUT4 in adipose tissue, skeletal muscle, and heart [33,34]. This is likely to be related to increased circulating free fatty acids which compete with glucose as energy substrate [35] and also inhibit the translocation of GLUT4 to the cell membrane [36].

Myocardial fatty acid uptake and utilization lend support to the hypothesis that myocardial insulin resistance is associated with cardiac dysfunction, characterized by increased left ventricle mass, reduction systolic function, and survival. Increasing fatty acid as fuel energy can lead to increased reactive oxygen species, thus contributing to structural and functional damage in the myocardium [37].

Accordingly, 9-week-old ZO rats show diastolic dysfunction with preserved ejection fraction [33]. These abnormalities occur prior to the onset of hypertension, which becomes elevated above control levels around 12 weeks of age [34]. Furthermore, cardiovascular complications similar to human obesity also include higher resting sympathetic nerve activity and reduced heart rate variability $[35,36]$ which were observed in $\mathrm{ZO}$ rats-attenuated baroreflexmediated changes in sympathetic nerve activity to vascular targets [32]. These are due to impairments in sympathetic and parasympathetic control of the heart $[37,38]$.

1.1.5. Zucker Diabetic Fatty Rats (fa/fa). Zucker Diabetic Fatty Rats (ZDF) phenotype originated from selective
TABLE 3: Main cardiovascular findings in diet-induced animal models of metabolic syndrome.

\begin{tabular}{ll}
\hline Models & Cardiovascular changes \\
\hline & (i) High blood pressure levels \\
& (ii) High heart rate \\
& (iii) Increased blood pressure variability \\
Fructose overload & (iv) Increased sympathetic modulation to the \\
& vessels and heart \\
& (v) Vascular oxidative stress \\
& (vi) Changes in left ventricular morphometry \\
& (vii) Diastolic dysfunction \\
& (viii) Increased cardiac effort \\
\hline (i) High blood pressure levels \\
(ii) High heart rate \\
(iii) Diastolic function \\
(iv) Systolic dysfunction \\
(v) Alterations in myocardial structure due to \\
reduction of calcium uptake in \\
sarcoplasmic reticulum of cardiomyocytes
\end{tabular}

breeding of Zucker rats with high glucose levels, which developed diabetes after 10 weeks of age. ZDF presents hyperphagia, as a result of a nonfunctioning leptin receptor, which in turn leads to obesity similar to the prediabetic state in humans [39]. Furthermore, hyperglycemia in the ZDF model is different from that observed in the ZO; ZDF rats do not have sufficient pancreatic $\beta$-cell function.

It has been observed that changes in left ventricular chamber morphology occurred in the untreated ZDF animals as early as 16 weeks of age [40]. Using positron emission tomography and echocardiography, van den Brom et al. (2009) found an increase in myocardial fatty acid oxidation, a reduction in insulin-mediated myocardial glucose utilization, associated with impairment of myocardial function in ZDF rats with 14 weeks of age [41]. In addition, vascular and neural dysfunction observed in ZDF rats with 12 weeks of age has been improved with vasopeptidase inhibitors [42].

In addition, this animal model shows an increase in plasma angiotensin converting enzyme activity and angiotensin II levels that, associated with high glucose levels, can lead to advanced nephropathy. This may be the reason by which ZDF rats started to die at 50 weeks of age ( 63 weeks for the control group), in association with an abrupt increase in blood urea nitrogen, suggesting that the cause of death was renal insufficiency [43]. 
1.1.6. DahlS.Z-Lepr ${ }^{f a} /$ Lepr $^{f a}$ Rat. One of the most recently created MS models is the DahlS.Z-Lepr ${ }^{\text {fa }} /$ Lepr $^{\text {fa }}$ rat (DS/obese). This MS model was established by crossing Dahl Salt rats and Zucker rats with the missense mutation in the leptin receptor gene (Lepr). The animals, once fed with a normal diet, developed obesity, as well as hypertension, dyslipidemia, insulin resistance, and type 2 diabetes. In addition, these animals developed cardiac hypertrophy, as well as renal and liver damage, which may account for their premature death. Hattori et al. (2011) showed that in an experimental period of 18 weeks, 13 (65\%) of 20 DS/obese rats died (seven from renal failure, two from cerebrovascular events, and four from sudden cardiac death); there were no deaths in the control group [44]. In addition, Murase et al. (2012) have recently demonstrated that body weight, as well as visceral and subcutaneous fat mass, was significantly increased in DS/obese female rats, which was associated with diastolic dysfunction and marked left ventricle hypertrophy and fibrosis [45]. Myocardial oxidative stress and inflammation, serum insulin, and triglyceride were also increased in DS/obese rats compared with DS/lean rats.

1.1.7. Otsuka Long-Evans Tokushima Fatty Rats. Otsuka Long-Evans Tokushima Fatty (OLETF) rats are a cholecystokinin 1 receptor knockout model which become obese secondarily to hyperphagia [46]. The increased food intake is characterized by a large increase in meal size with a decrease in meal frequency which is not sufficient to compensate for the meal size increase. As a result, these animals usually present hyperglycemia after 18 weeks of age, mild obesity, and diabetes mellitus, more frequently observed in males [47]. However, insulin resistance in OLETF rats emerges at 12 weeks of age, before the impairment of pancreatic $\beta$-cell function [48].

Mizushige et al. (2000) have previously demonstrated that OLETF rats present diastolic dysfunction in their prediabetic state (15 weeks), observed by a prolongation in deceleration time and a decrease in amplitude of peak velocity of the early diastolic filling wave, without changes in the blood pressure and heart rate. In addition, the researchers observed extracellular fibrosis and abundant transforming growth factor- $\beta 1$ receptor II in the left ventricle of these rats [49]. While still in the prediabetic stage, OLETF rats exhibited a lower coronary flow reserve and increased coronary vascular resistance during hyperemia, which was associated with increased wallto-lumen ratio and perivascular fibrosis [50]. In the late stage of diabetes (22 and 62 weeks of age), it was demonstrated that these animals displayed an impairment in diastolic function and changes in the geometry of conductance and resistance arteries [51].

1.1.8. Goto-Kakizaki Rats. The Goto-Kakizaki (GK) model was created by selective breeding of an outbred colony of Wistar rats, selected for high glucose levels in an oral glucose tolerance test [52]. These animals develop hyperglycemia after 4 weeks of age [53] and increased liver and plasma lipid concentration after 8 weeks of age [54].
After 16 weeks of age, at prediabetic state, GK rats displayed left ventricle remodeling with marked hypertrophy of cardiomyocytes and increased extracellular matrix deposition, culminating in increased heart size [55]. Despite the progressively worsening of glucose metabolism derangement, at 18 months of age the contractile function of the heart appears to be well preserved, as observed by maintenance of amplitude of shortening in electrically stimulated myocytes [56]. In addition, untreated GK rats presented mild hypertension and a blunted vascular relaxation by acetylcholine and sodium nitroprusside when compared to control animals [57].

\subsection{Chemically Induced Models}

1.2.1. Monosodium Glutamate-Induced Spontaneously Hypertensive Rat. We studied in our laboratory the cardiovascular autonomic function of obesity induced by monosodium glutamate (MSG) in a normotensive model. Male Wistar rats receiving MSG neonatal treatment showed metabolic abnormalities, as well as increased body weight, Lee index, epididymal white adipose tissue, and insulin resistance. In addition, these animals exhibited reduced glucose/insulin index $(-62.5 \%)$, and increased insulin secretion during glucose overload (39.3\%), and hyperinsulinemia. These rats showed a slight increase in mean arterial pressure with no difference in the heart rate from their controls. However, they showed cardiovascular autonomic dysfunction, as shown by reduced baroreflex sensitivity and vagal and sympathetic effects when compared to their controls. We observed a reduced sympathetic effect which was not followed by changes in the basal heart rate or tachycardic responses to arterial pressure changes [58].

Recently, we carried out studies on cardiovascular abnormalities of the spontaneously hypertensive rat (SHR) $[59,60]$ treated with MSG in the neonatal period [61]. Although this animal model is not new $[62,63]$, no research, to our knowledge, has been undertaken using metabolic and cardiovascular parameters over time $(3,6$, and 9 months of age). The use of MSG in genetically hypertensive rats led these animals to progressively increase body adiposity and triglyceride levels. Besides developing and maintaining insulin resistance, they presented low HDL cholesterol and increased inflammation state (high C-reactive protein, interleukin 6 , tumor necrosis factor- $\alpha$ levels, and low adiponectin levels), which reached highest levels at 6 and 9 months of age [61].

In MS, hypertension is commonly associated with metabolic changes of the syndrome. Therefore, the use of a genetically hypertensive rats associated with MSG treatment, which leads to metabolic changes including obesity, seems to work well as an MS model resembling the human disease. However, early studies showed that SHR treated with MSG did not maintain hypertension [62], but it should be noted that indirect tail-cuff methods were used. Thus, we evaluated blood pressure by direct method (analyzed on a beat-to-beat basis) at 3, 6, and 9 months in MSG-treated SHR compared to SHR and normotensive rats [61]. It was observed that 
mean blood pressure was similarly higher in SHR and MSGtreated SHR at all ages, when compared to normotensive rats. Furthermore, there were no changes in blood pressure over time in the groups studied.

The increased cardiovascular mortality which characterizes MS may be partially attributed to cardiac sympathovagal imbalance $[64,65]$. Thus, we evaluated temporal cardiovascular autonomic dysfunction in this model by spectral analysis and observed that the changes in cardiovascular autonomic control evaluated up to 9 months are similar to those observed in hypertensive rats. At 9 months the animals maintained the hypertensive state, a feature of SHR, and presented reduced heart rate variability, decreased spontaneous baroreflex sensibility, and increased cardiac sympathovagal balance. In addition to these changes, it was observed that SHR and SHR with MSG-induced obesity showed increments of 6- to 8-fold of systolic arterial pressure variability at 6 and 9 months, as compared to normotensive rats. Interestingly, at 6 months, MSG-induced SHR had increased systolic arterial pressure variability when compared to only SHR. Taken together, SHRs with MSG-induced obesity show an impairment of the cardiovascular nervous control. Sympathetic activation plays an important role in the pathogenesis of insulin resistance [66-68] and in the activation of the reninangiotensin system [69], which are related to cardiovascular autonomic control dysfunction of MS [64].

1.2.2. Streptozotocin Administration. The injection of streptozotocin (STZ) to mice, rats, and rabbits has been widely used as a model of type 1 diabetes. Streptozotocin destroys pancreatic $\beta$ cells, resulting in a diabetic syndrome in animals, characterized by hyperglycemia, hypoinsulinemia, glycosuria, and body weight loss [70, 71]. These rats have systolic and diastolic dysfunction at rest, as evaluated by left ventricular catheterization and echocardiography, as well as reduced capacity for cardiac adjustment to volume overload and maximal oxygen consumption [72, 73]. Furthermore, STZ induces reduction in baroreflex-mediated bradycardia and tachycardia, as well as impairment in cardiac vagal tone in the face of unaltered sympathetic tone [71, 74, 75].

Although well accepted in the literature as a model for type 1 diabetes, the injection of STZ alone does not allow it to mimic the characteristics of MS in humans, since these animals show hypoinsulinemia, loss or no change in body weight, and hypotension $[71,74,75]$. An alternative approach, previously used, is the administration of low doses of STZ $(25 \mathrm{mg} / \mathrm{Kg})$ associated with high fructose diet in rats. This combination induced mild hyperglycemia and hypertriglyceridemia associated with mild fasting hyperinsulinemia and whole body insulin resistance, without significant increase in body weight [76]. In addition, the authors also observed decreased left ventricular contractile function and reduced myocardial metabolic efficiency.

\subsection{Diet-Induced Models}

1.3.1. Fructose Overload. Since 1978 the mean daily intake of added fructose and total fructose has increased in both sexes and all age groups, having as the main sources of consumption soft drinks and other sweetened beverages $[77,78]$. Fructose overload in drinking water or chow has been used to promote metabolic, hemodynamic, structural, and functional derangements in rodents. Fructose overload in experimental animals has been associated with high triglyceride levels, adiposity, insulin resistance, and glucose intolerance [7986]. These animals have been used by our group in order to understand the various aspects of obesity, dyslipidemia, and insulin resistance-associated cardiovascular changes [81-88]. We observed that an increased sympathetic modulation to the vessels and heart preceded metabolic dysfunction in fructoseconsuming mice, thus suggesting that changes in autonomic modulation may be a triggering mechanism underlying the cluster of symptoms associated with cardiometabolic disease [83-86, 88].

Animal studies have shown strong associations between high fructose intake and the onset of arterial hypertension. According to Farah et al., mice which received a high fructose diet showed higher blood pressure and heart rate in the dark (active) period when compared with the light (resting) period [87]. This augment in blood pressure was related to an increase in the blood pressure variability and, consequently, to increased vascular sympathetic modulation. This is a relevant finding since during the dark period the mice are active (grooming, eating, and drinking) and the sympathetic activity should be high. On the other hand, it was displayed that female fructose-fed rats presented reduced vagal tonus, unaltered sympathetic tonus, and intrinsic heart rate [81]. However, this study also showed that although the sympathetic tonus remained unchanged, the autonomic balance (sympathetic/parasympathetic) was altered, leading to a sympathetic predominance. A positive correlation between reduced cardiac vagal tonus and insulin resistance was also found, thus reinforcing the relationship between autonomic and metabolic dysfunction [81].

Since arterial baroreflex influences both sympathetic and parasympathetic outflow, disorders in autonomic neuronal pathways (efferent or afferent) may affect cardiovascular health and be related to high blood pressure. High fructose consumption induces an impairment of baroreflex sensitivity, as evaluated by heart rate responses associated with arterial pressure changes induced by vasoactive drugs $[81,86]$, as well as by linear regression and alpha-index [84]. Furthermore, unpublished data from our laboratory demonstrated that impairment of baroreflex sensitivity was positively correlated with the number of elastic lamellae in the ascendant aorta. This data suggest that the possible loss of distensibility of the aorta may be associated with changes in baroreflex sensitivity, since the mechanical stress of the arterial wall cannot be effective to properly trigger the mechanoreceptors.

Regarding the rennin-angiotensin system, it has been widely acknowledged that high-fructose diet induces increased angiotensin II plasma levels, which contributes to hypertension, insulin resistance, and dyslipidemia [89], and may account for cardiac remodeling $[80,90]$ and vascular oxidative stress [91] in this MS model. In fact, it appears that angiotensin II promotes fibroblast proliferation due to activation of the angiotensin II type 1 receptor, which results 
in increased collagen type III expression and accumulation in the heart [92]. Also, there is evidence that the insulinresistant state is associated with angiotensin II type 1 receptor upregulation and increased endothelial (aorta) superoxide anion levels, which is likely to be caused by an increase in $\mathrm{NAD}(\mathrm{P}) \mathrm{H}$ oxidase expression in fructose-fed rats [91]. Furthermore, it should be stressed that angiotensin la receptors are critical in mediating the response to a highfructose diet and the resultant state of glucose intolerance, because in the absence of these receptors, a fructose diet decreases the blood pressure, as observed in angiotensin la knockout mice [87].

As an important consequence of these metabolic, hemodynamic, autonomic, and structural changes displayed in this MS model, our group recently demonstrated that fructose overload promoted changes in left ventricular morphometry, diastolic dysfunction, and increased cardiac effort, as evidenced by the increase in the myocardial performance index [85]. Other researchers have found similar results for Wistar rats receiving a $10 \%$ fructose overload for 8 weeks [93].

1.3.2. Sucrose Overload. Sucrose is a disaccharide composed by one molecule of glucose linked to one molecule of fructose through an $\alpha 1-4$ glycoside bond [94]. Similar to fructose, sucrose induces MS in animals, as increased plasma concentrations of insulin, leptin, triglycerides, glucose, and free fatty acids and impaired glucose tolerance were shown [95]. Sucrose-treated rats (32\% in drinking water) revealed early abnormalities in diastolic function ( 2.5 weeks of treatment) followed by late systolic dysfunction and concurrent alterations in myocardial structure (10 weeks of treatment). Furthermore, the authors demonstrated that after 10 weeks of sucrose treatment the animals presented reduced calcium uptake in sarcoplasmic reticulum of cardiomyocytes [96].

In addition, animals treated with an $8 \%$ sucrose solution developed hypertension and tachycardia after 2 weeks of treatment, and this was not related to weight gain. The authors concluded that sucrose ingestion may stimulate the ventromedial hypothalamus to increase sympathetic activity and elevate blood pressure in rats [97].

1.3.3. High Fat Diet. High fat diets have been used with fat fractions between $20 \%$ and $60 \%$ energy as fat, and the basic fat component varies between animal-derived fats and plant oils, for example, corn, coconut, or safflower oil [98]. After 10 weeks of a high fat diet, rats displayed high fat mass, insulin resistance, and hyperleptinemia, typically associated with obesity [99]. Rabbits receiving a high fat diet during 3 weeks presented an impairment in leptin sensibility, along with increased mean arterial pressure, heart rate, and plasma norepinephrine concentration. Renal sympathetic nerve activity was also higher in high fat diet rabbits when compared to control diet rabbits and was correlated to plasma leptin [100]. Furthermore, Fardin et al. (2012) have demonstrated that the baroreceptor dysfunction which controls renal sympathetic nerve activity is an initial change in the obesity-induced high fat-fed rats, which might be a predictor of sympathoexcitation and hypertension associated with obesity [101].
The renin-angiotensin system has been involved in the hypertension genesis linked to obesity [102]. In fact, Boustany et al. (2004) observed that both angiotensinogen gene expressions in retroperitoneal fat mass and plasma angiotensinogen concentration were increased in rats receiving high fat diet, thus showing increased activity of the adipose and systemic rennin-angiotensin system in obesity-related hypertension [103].

1.3.4. Cafeteria Diet. Proponents of chemically induced MS models have been challenged by researchers who argue for diet-induced models. The latter contend that these reflect more accurately the condition of human obesity when compared to chemically induced or genetic modifications [104].

Thus, several diet-induced experimental models have been proposed, suggesting that there is an aggregation of factors in MS. The cafeteria diet model [105] is a fine example of a diet-induced experimental model. In this model, animals are allowed free access to standard chow and water while concurrently given highly palatable, energy dense, unhealthy human foods ad libitum, which promotes voluntary hyperphagia. This diet results in weight gain, increased fat mass, glucose intolerance, and insulin resistance $[106,107]$.

Wistar rats fed a cafeteria diet showed, in addition to the classical metabolic disorder, cardiovascular alterations, such as increased heart rate and blood pressure [108]. Cafeteria diet has been shown to lead to impairment of the endothelium-derived hyperpolarization mechanism, in particular, potassium channel signaling mechanisms [109]. Thus, this finding may explain, at least in part, the link between cafeteria diet and increased blood pressure in rats; this link is further reinforced by understanding that vascular tone refers to the balance between constrictor and dilator actions and influences the control of blood flow and pressure, and this phenomenon is influenced by endothelium-derived hyperpolarization mechanism.

Although normotensive rats fed a cafeteria diet showed an increase in blood pressure, this diet may have a better outcome if used in SHR, since this animal model has cardiac disorders, for example, increased sympathetic activity, hypertension, and cardiac hypertrophy $[59,60]$ which may be potentiated by the cafeteria diet. Spontaneously hypertensive rats fed with cafeteria diet for 12 weeks showed metabolic changes similar to those of the MS, for example, high plasma levels of glycemia, insulin, triglyceride, leptin, and obesity. Furthermore, animals maintained hypertension, a common feature of SHR [110]. Many studies have dealt with the autonomic dysfunction of SHR by spectral analysis [111, 112].

\section{Conclusions}

There is considerable evidence to support the hypothesis that the cluster of complications from metabolic syndrome status converges to a derangement of the cardiovascular system. In fact, cardiovascular diseases are the leading cause of morbidity and mortality in this condition. The use of different experimental models that mimic the metabolic derangements seen in this syndrome is extremely important in order to 
better understand the mechanisms involved in cardiovascular changes caused by metabolic syndrome. New approaches, such as genetically modified models, can provide mechanistic information on the genetic and environmental factors involved in the development of cardiovascular dysfunction in the metabolic syndrome.

\section{References}

[1] K. G. M. M. Alberti, P. Zimmet, and J. Shaw, "Metabolic syndrome-a new world-wide definition. A consensus statement from the International Diabetes Federation," Diabetic Medicine, vol. 23, no. 5, pp. 469-480, 2006.

[2] L. F. Defina, G. L. Vega, D. Leonard, and S. M. Grundy, "Fasting glucose, obesity, and metabolic syndrome as predictors of type 2 diabetes: the cooper center longitudinal study," Journal of Investigative Medicine, vol. 60, no. 8, pp. 1164-1168, 2012.

[3] J. M. Dekker, C. Girman, T. Rhodes et al., "Metabolic syndrome and 10-year cardiovascular disease risk in the Hoorn Study," Circulation, vol. 112, no. 5, pp. 666-673, 2005.

[4] G. Mulè, E. Nardi, S. Cottone et al., "Influence of metabolic syndrome on hypertension-related target organ damage," Journal of Internal Medicine, vol. 257, no. 6, pp. 503-513, 2005.

[5] L. de las Fuentes, A. L. Brown, S. J. Mathews et al., "Metabolic syndrome is associated with abnormal left ventricular diastolic function independent of left ventricular mass," European Heart Journal, vol. 28, no. 5, pp. 553-559, 2007.

[6] M. Pyörälä, H. Miettinen, P. Halonen, M. Laakso, and K. Pyörälä, "Insulin resistance syndrome predicts the risk of coronary heart disease and stroke in healthy middle-aged men: the 22-year follow-up results of the Helsinki Policemen Study," Arteriosclerosis, Thrombosis, and Vascular Biology, vol. 20, no. 2, pp. 538-544, 2000.

[7] K. M. Choi, O. H. Ryu, K. W. Lee et al., "Serum adiponectin, interleukin-10 levels and inflammatory markers in the metabolic syndrome," Diabetes Research and Clinical Practice, vol. 75, no. 2, pp. 235-240, 2007.

[8] F. Emanuela, M. Grazia, D. R. Marco, L. Maria Paola, F. Giorgio, and B. Marco, "Inflammation as a link between obesity and metabolic syndrome," Journal of Nutrition and Metabolism, vol. 2012, Article ID 476380, 7 pages, 2012.

[9] H. Chen, O. Charlat, L. A. Tartaglia et al., "Evidence that the diabetes gene encodes the leptin receptor: identification of a mutation in the leptin receptor gene in $\mathrm{db} / \mathrm{db}$ mice," Cell, vol. 84, no. 3, pp. 491-495, 1996.

[10] N. Kanie and K. Kamata, "Contractile responses in spontaneously diabetic mice. I. Involvement of superoxide anion in enhanced contractile response of aorta to norepinephrine in C57BL/KsJ(db/db) mice," General Pharmacology, vol. 35, no. 6, pp. 311-318, 2000.

[11] D. Senador, K. Kanakamedala, M. C. Irigoyen, M. Morris, and K. M. Elased, "Cardiovascular and autonomic phenotype of $\mathrm{db} / \mathrm{db}$ diabetic mice," Experimental Physiology, vol. 94, no. 6, pp. 648-658, 2009.

[12] F. Tessari, R. A. Travagli, R. Zanoni, and M. Prosdocimi, "Effects of long-term diabetes and treatment with gangliosides on cardiac sympathetic innervation: a biochemical and functional study in mice," Journal of Diabetic Complications, vol. 2, no. 1, pp. 34-37, 1988.

[13] L. M. Semeniuk, A. J. Kryski, and D. L. Severson, "Echocardiographic assessment of cardiac function in diabetic $\mathrm{db} / \mathrm{db}$ and transgenic db/db-hGLUT4 mice," American Journal of Physiology, vol. 283, no. 3, pp. H976-H982, 2002.

[14] A. Daniels, M. van Bilsen, B. J. A. Janssen et al., "Impaired cardiac functional reserve in type 2 diabetic $\mathrm{db} / \mathrm{db}$ mice is associated with metabolic, but not structural, remodelling," Acta Physiologica, vol. 200, no. 1, pp. 11-22, 2010.

[15] H. Iwatsuka, A. Shino, and Z. Suzuoki, "General survey of diabetic features of yellow KK mice," Endocrinologia Japonica, vol. 17, no. 1, pp. 23-35, 1970.

[16] M. Aizawa-Abe, Y. Ogawa, H. Masuzaki et al., "Pathophysiological role of leptin in obesity-related hypertension," The Journal of Clinical Investigation, vol. 105, no. 9, pp. 1243-1252, 2000.

[17] K. Ohashi, S. Kihara, N. Ouchi et al., "Adiponectin replenishment ameliorates obesity-related hypertension," Hypertension, vol. 47, no. 6, pp. 1108-1116, 2006.

[18] Y. Zhang, R. Proenca, M. Maffei, M. Barone, L. Leopold, and J. M. Friedman, "Positional cloning of the mouse obese gene and its human homologue," Nature, vol. 372, no. 6505, pp. 425-432, 1994.

[19] A. L. Mark, R. A. Shaffer, M. L. G. Correia, D. A. Morgan, C. D. Sigmund, and W. G. Haynes, "Contrasting blood pressure effects of obesity in leptin-deficient ob/ob mice and agouti yellow obese mice," Journal of Hypertension, vol. 17, no. 12, part 2, pp. 1949-1953, 1999.

[20] J. B. Young and L. Landsberg, "Diminished sympathetic nervous system activity in genetically obese (ob/ob) mouse," American Journal of Physiology, vol. 245, no. 2, pp. E148-E154, 1983.

[21] S. J. Swoap, "Altered leptin signaling is sufficient, but not required, for hypotension associated with caloric restriction," American Journal of Physiology, vol. 281, no. 6, pp. H2473H2479, 2001

[22] P. Dobrzyn, A. Dobrzyn, M. Miyazaki, and J. M. Ntambi, "Loss of stearoyl-CoA desaturase 1 rescues cardiac function in obese leptin-deficient mice," Journal of Lipid Research, vol. 51, no. 8, pp. 2202-2210, 2010.

[23] A. K. M. T. Zaman, S. Fujii, D. Goto et al., "Salutary effects of attenuation of angiotensin II on coronary perivascular fibrosis associated with insulin resistance and obesity," Journal of Molecular and Cellular Cardiology, vol. 37, no. 2, pp. 525-535, 2004.

[24] S. Ae Park, M. S. Choi, S. Y. Cho et al., "Genistein and daidzein modulate hepatic glucose and lipid regulating enzyme activities in C57BL/KsJ-db/db mice," Life Sciences, vol. 79, no. 12, pp. 12071213, 2006.

[25] Y. F. Dong, L. Liu, K. Kataoka et al., "Aliskiren prevents cardiovascular complications and pancreatic injury in a mouse model of obesity and type 2 diabetes," Diabetologia, vol. 53, no. 1, pp. 180-191, 2010.

[26] J. van den Brandt, P. Kovacs, and I. Kloting, "Features of the metabolic syndrome in the spontaneously hypertriglyceridemic Wistar Ottawa Karlsburg W (RT1 ${ }^{u}$ haplotype) rat," Metabolism, vol. 49, no. 9, pp. 1140-1144, 2000.

[27] J. van den Brandt, P. Kovacs, and I. Kloting, "Metabolic features in disease-resistant as well as in spontaneously hypertensive rats and newly established obese Wistar Ottawa Karlsburg inbred rats," International Journal of Obesity, vol. 24, no. 12, pp. 1618$1622,2000$.

[28] H. Kondo, L. Shimomura, Y. Matsukawa et al., "Association of adiponectin mutation with type 2 diabetes: a candidate gene for the insulin resistance syndrome," Diabetes, vol. 51, no. 7, pp. 2325-2328, 2002. 
[29] O. Grisk, T. Frauendorf, T. Schlüter et al., "Impaired coronary function in Wistar Ottawa Karlsburg W rats-a new model of the metabolic syndrome," Pflügers Archiv, vol. 454, no. 6, pp. 1011-1021, 2007.

[30] L. M. Davis, M. Michaelides, L. J. Cheskin et al., "Bromocriptine administration reduces hyperphagia and adiposity and differentially affects dopamine D2 receptor and transporter binding in leptin-receptor-deficient Zucker rats and rats with dietinduced obesity," Neuroendocrinology, vol. 89, no. 2, pp. 152-162, 2009.

[31] L. M. Zucker and H. N. Antoniades, "Insulin and obesity in the Zucker genetically obese rat 'fatty"' Endocrinology, vol. 90, no. 5, pp. 1320-1330, 1972.

[32] A. M. Schreihofer, D. A. Mandel, S. C. Mobley, and D. W. Stepp, "Impairment of sympathetic baroreceptor reflexes in obese Zucker rats," American Journal of Physiology, vol. 293, no. 4, pp. H2543-H2549, 2007.

[33] X. Zhou, L. Ma, J. Habibi et al., "Nebivolol improves diastolic dysfunction and myocardial remodeling through reductions in oxidative stress in the Zucker obese rat," Hypertension, vol. 55, no. 4, pp. 880-888, 2010.

[34] J. M. Osmond, J. D. Mintz, B. Dalton, and D. W. Stepp, “Obesity increases blood pressure, cerebral vascular remodeling, and severity of stroke in the Zucker rat," Hypertension, vol. 53, no. 2, pp. 381-386, 2009.

[35] D. A. Morgan, E. A. Anderson, and A. L. Mark, "Renal sympathetic nerve activity is increased in obese Zucker rats," Hypertension, vol. 25, no. 4, part 2, pp. 834-838, 1995.

[36] J. M. Overton, T. D. Williams, J. B. Chambers, and M. E. Rashotte, "Cardiovascular and metabolic responses to fasting and thermoneutrality are conserved in obese Zucker rats," American Journal of Physiology, vol. 280, no. 4, pp. R1007-R1015, 2001.

[37] R. D. Bunag and D. L. Barringer, "Obese Zucker rats, though still normotensive, already have impaired chronotropic baroreflexes," Clinical and Experimental Hypertension A, vol. 10, no. 1, supplement 1, pp. 257-262, 1988.

[38] D. L. Barringer and R. D. Bunag, "Uneven blunting of chronotropic baroreflexes in obese Zucker rats," American Journal of Physiology, vol. 256, no. 2, part 2, pp. H417-H421, 1989.

[39] S. A. Marsh, P. C. Powell, A. Agarwal, L. J. Dell'Italia, and J. C. Chatham, "Cardiovascular dysfunction in Zucker obese and Zucker diabetic fatty rats: role of hydronephrosis," American Journal of Physiology, vol. 293, no. 1, pp. H292-H298, 2007.

[40] J. Baynes and D. B. Murray, "Cardiac and renal function are progressively impaired with aging in Zucker diabetic fatty type II diabetic rats," Oxidative Medicine and Cellular Longevity, vol. 2, no. 5, pp. 328-334, 2009.

[41] C. E. van den Brom, M. C. Huisman, R. Vlasblom et al., "Altered myocardial substrate metabolism is associated with myocardial dysfunction in early diabetic cardiomyopathy in rats: studies using positron emission tomography," Cardiovascular Diabetology, vol. 8, article 39, 2009.

[42] C. L. Oltman, E. P. Davidson, L. J. Coppey, T. L. Kleinschmidt, and M. A. Yorek, "Treatment of Zucker diabetic fatty rats with AVE7688 improves vascular and neural dysfunction," Diabetes, Obesity and Metabolism, vol. 11, no. 3, pp. 223-233, 2009.

[43] M. Mizuno, T. Sada, M. Kato, Y. Fukushima, H. Terashima, and H. Koike, "The effect of angiotensin II receptor blockade on an end-stage renal failure model of type 2 diabetes," Journal of Cardiovascular Pharmacology, vol. 48, no. 4, pp. 135-142, 2006.
[44] T. Hattori, T. Murase, M. Ohtake et al., "Characterization of a new animal model of metabolic syndrome: the DahlS.ZLepr(fa)/Lepr(fa) rat," Nutrition \& Diabetes, vol. 1, no. 1, pp. ele6, 2011.

[45] T. Murase, T. Hattori, M. Ohtake et al., "Cardiac remodeling and diastolic dysfunction in DahlS.Z-Lepr(fa)/Lepr(fa) rats: a new animal model of metabolic syndrome," Hypertension Research, vol. 35, no. 2, pp. 186-193, 2012.

[46] T. H. Moran, "Unraveling the obesity of OLETF rats," Physiology and Behavior, vol. 94, no. 1, pp. 71-78, 2008.

[47] K. Kawano, T. Hirashima, S. Mori, and T. Natori, "OLETF (Otsuka Long-Evans Tokushima Fatty) rat: a new NIDDM rat strain," Diabetes Research and Clinical Practice, vol. 24, supplement, pp. S317-S320, 1994.

[48] T. Sato, Y. Asahi, K. Toide, and N. Nakayama, "Insulin resistance in skeletal muscle of the male Otsuka Long-Evans Tokushima Fatty rat, a new model of NIDDM," Diabetologia, vol. 38, no. 9, pp. 1033-1041, 1995.

[49] K. Mizushige, L. Yao, T. Noma et al., "Alteration in left ventricular diastolic filling and accumulation of myocardial collagen at insulin-resistant prediabetic stage of a type II diabetic rat model," Circulation, vol. 101, no. 8, pp. 899-907, 2000.

[50] Y. Yu, K. Ohmori, I. Kondo et al., "Correlation of functional and structural alterations of the coronary arterioles during development of type II diabetes mellitus in rats," Cardiovascular Research, vol. 56, no. 2, pp. 303-311, 2002.

[51] F. Saito, M. Kawaguchi, J. Izumida, T. Asakura, K. Maehara, and Y. Maruyama, "Alteration in haemodynamics and pathological changes in the cardiovascular system during the development of type 2 diabetes mellitus in OLETF rats," Diabetologia, vol. 46, no. 8, pp. 1161-1169, 2003.

[52] Y. Goto, M. Kukizaki, and N. Masaki, "Spontaneous diabetes produced by selective breeding of normal Wistar rats," Proceedings of the Japan Academy, vol. 51, no. 1, pp. 80-85, 1975.

[53] S. Gupte, N. Labinskyy, R. Gupte, A. Csiszar, Z. Ungvari, and J. G. Edwards, "Role of $\mathrm{NAD}(\mathrm{P}) \mathrm{H}$ oxidase in superoxide generation and endothelial dysfunction in Goto-Kakizaki (GK) rats as a model of nonobese NIDDM," PLoS ONE, vol. 5, no. 7, Article ID el1800, 2010.

[54] S. Akiyama, S. I. Katsumata, K. Suzuki, Y. Nakaya, Y. Ishimi, and M. Uehara, "Hypoglycemic and hypolipidemic effects of hesperidin and cyclodextrin-clathrated hesperetin in GotoKakizaki rats with type 2 diabetes," Bioscience, Biotechnology and Biochemistry, vol. 73, no. 12, pp. 2779-2782, 2009.

[55] A. D’Souza, F. C. Howarth, J. Yanni et al., "Left ventricle structural remodelling in the prediabetic Goto-Kakizaki rat," Experimental Physiology, vol. 96, no. 9, pp. 875-888, 2011.

[56] F. C. Howarth, M. Shafiullah, and M. A. Qureshi, "Chronic effects of type 2 diabetes mellitus on cardiac muscle contraction in the Goto-Kakizaki rat," Experimental Physiology, vol. 92, no. 6, pp. 1029-1036, 2007.

[57] K. Witte, I. Reitenbach, K. Stolpe, L. Schilling, M. Kirchengast, and B. Lemmer, "Effects of the endothelin a receptor antagonist darusentan on blood pressure and vascular contractility in type 2 diabetic Goto-Kakizaki rats," Journal of Cardiovascular Pharmacology, vol. 41, no. 6, pp. 890-896, 2003.

[58] S. P. Konrad, V. Farah, B. Rodrigues et al., "Monosodium glutamate neonatal treatment induces cardiovascular autonomic function changes in rodents," Clinics, vol. 67, no. 10, pp. 12091214, 2012. 
[59] J. M. Pfeffer, M. A. Pfeffer, M. C. Fishbein, and E. D. Frohlich, "Cardiac function and morphology with aging in the spontaneously hypertensive rat," American Journal of Physiology, vol. 237, no. 4, pp. H461-468, 1979.

[60] S. A. Doggrell and L. Brown, "Rat models of hypertension, cardiac hypertrophy and failure," Cardiovascular Research, vol. 39, no. 1, pp. 89-105, 1998.

[61] N. M. Leguisamo, A. M. Lehnen, U. F. Machado et al., "GLUT4 content decreases along with insulin resistance and high levels of inflammatory markers in rats with metabolic syndrome," Cardiovascular Diabetology, vol. 11, no. 1, p. 100, 2012.

[62] M. van den Buuse, D. H. G. Versteeg, and W. de Jong, "Effects of neonatal treatment with monosodium-glutamate in spontaneously hypertensive rats," Brain Research, vol. 351, no. 1, pp. 135-138, 1985.

[63] J. F. Lorden and A. Caudle, "Behavioral and endocrinological effects of single injections of monosodium glutamate in the mouse," Neurobehavioral Toxicology and Teratology, vol. 8, no. 5, pp. 509-519, 1986.

[64] G. Garruti, F. Giampetruzzi, M. G. Vita et al., "Links between metabolic syndrome and cardiovascular autonomic dysfunction," Experimental Diabetes Research, vol. 2012, no. 1, Article ID 615835, 9 pages, 2012.

[65] G. Grassi, F. Arenare, F. Quarti-Trevano, G. Seravalle, and G. Mancia, "Heart rate, sympathetic cardiovascular influences, and the metabolic syndrome," Progress in Cardiovascular Diseases, vol. 52, no. 1, pp. 31-37, 2009.

[66] T. Pollare, H. Lithell, I. Selinus, and C. Berne, "Application of prazosin is associated with an increase of insulin sensitivity in obese patients with hypertension," Diabetologia, vol. 31, no. 7, pp. 415-420, 1988.

[67] K. A. Jamerson, S. Julius, T. Gudbrandsson, O. Andersson, and D. O. Brant, "Reflex sympathetic activation induces acute insulin resistance in the human forearm," Hypertension, vol. 21, no. 5, pp. 618-623, 1993.

[68] S. Snitker, I. Macdonald, E. Ravussin, and A. Astrup, "The sympathetic nervous system and obesity: role in aetiology and treatment," Obesity Reviews, vol. 1, no. 1, pp. 5-15, 2000.

[69] J. R. Sowers, M. Nyby, and N. Stern, "Blood pressure and hormone changes associated with weight reduction in the obese," Hypertension, vol. 4, no. 5, pp. 686-691, 1982.

[70] A. Junod, A. E. Lambert, W. Stauffacher, and A. E. Renold, "Diabetogenic action of streptozotocin: relationship of dose to metabolic response," The Journal of Clinical Investigation, vol. 48, no. 11, pp. 2129-2139, 1969.

[71] K. C. Tomlinson, S. M. Gardiner, R. A. Hebden, and T. Bennett, "Functional consequences of streptozotocin-induced diabetes mellitus, with particular reference to the cardiovascular system," Pharmacological Reviews, vol. 44, no. 1, pp. 103-150, 1992.

[72] B. Rodrigues, D. M. Figueroa, C. T. Mostarda, M. V. Heeren, M. C. Irigoyen, and K. de Angelis, "Maximal exercise test is a useful method for physical capacity and oxygen consumption determination in streptozotocin-diabetic rats," Cardiovascular Diabetology, vol. 6, no. 1, article 38, 2007.

[73] R. Wichi, C. Malfitano, K. Rosa et al., "Noninvasive and invasive evaluation of cardiac dysfunction in experimental diabetes in rodents," Cardiovascular Diabetology, vol. 6, no. 1, article 14, 2007.

[74] A. D. Harthmann, K. de Angelis, L. P. Costa et al., "Exercise training improves arterial baro- and chemoreflex in control and diabetic rats," Autonomic Neuroscience, vol. 133, no. 2, pp. 115120, 2007.
[75] S. B. C. Souza, K. Flues, J. Paulini et al., "Role of exercise training in cardiovascular autonomic dysfunction and mortality in diabetic ovariectomized rats," Hypertension, vol. 50, no. 4, pp. 786-791, 2007.

[76] S. L. Ménard, E. Croteau, O. Sarrhini et al., "Abnormal in vivo myocardial energy substrate uptake in diet-induced type 2 diabetic cardiomyopathy in rats," American Journal of Physiology, vol. 298, no. 5, pp. E1049-E1057, 2010.

[77] L. Ferder, M. D. Ferder, and F. Inserra, "The role of high-fructose corn syrup in metabolic syndrome and hypertension," Current Hypertension Reports, vol. 12, no. 2, pp. 105-112, 2010.

[78] R. A. Forshee, P. A. Anderson, and M. L. Storey, "Sugarsweetened beverages and body mass index in children and adolescents: a meta-analysis," American Journal of Clinical Nutrition, vol. 87, no. 6, pp. 1662-1671, 2008.

[79] F. J. Martinez, R. A. Rizza, and J. C. Romero, "High-fructose feeding elicits insulin resistance, hyperinsulinism, and hypertension in normal mongrel dogs," Hypertension, vol. 23, no. 4, pp. 456-463, 1994.

[80] K. Kamide, H. Rakugi, J. Higaki et al., “The renin-angiotensin and adrenergic nervous system in cardiac hypertrophy in fructose-fed rats," American Journal of Hypertension, vol. 15, no. 1, part 1, pp. 66-71, 2002.

[81] J. O. Brito, K. Ponciano, D. Figueroa et al., "Parasympathetic dysfunction is associated with insulin resistance in fructosefed female rats," Brazilian Journal of Medical and Biological Research, vol. 41, no. 9, pp. 804-808, 2008.

[82] T. S. Cunha, V. Farah, J. Paulini et al., "Relationship between renal and cardiovascular changes in a murine model of glucose intolerance," Regulatory Peptides, vol. 139, no. 1-3, pp. 1-4, 2007.

[83] K. de Angelis, D. D. Senador, C. Mostarda, M. C. Irigoyen, and M. Morris, "Sympathetic overactivity precedes metabolic dysfunction in a fructose model of glucose intolerance in mice," American Journal of Physiology, vol. 302, no. 8, pp. R950-R957, 2012.

[84] J. F. Machi, N. Bernardes, C. Mostarda et al., "Walking promotes metabolic and baroreflex sensitivity improvement in fructosefed male rats," European Journal of Applied Physiology, vol. 113, no. 1, pp. 41-49, 2012.

[85] C. Mostarda, I. C. Moraes-Silva, V. M. Salemi et al., "Exercise training prevents diastolic dysfunction induced by metabolic syndrome in rats," Clinics, vol. 67, no. 7, pp. 815-820, 2012.

[86] I. C. Sanches, J. de Oliveira Brito, G. O. Candido et al., "Cardiometabolic benefits of exercise training in an experimental model of metabolic syndrome and menopause," Menopause, vol. 19, no. 5, pp. 562-568, 2012.

[87] V. Farah, K. M. Elased, Y. Chen et al., "Nocturnal hypertension in mice consuming a high fructose diet," Autonomic Neuroscience, vol. 130, no. 1-2, pp. 41-50, 2006.

[88] D. Senador, S. Shewale, M. C. Irigoyen, K. M. Elased, and M. Morris, "Effects of restricted fructose access on body weight and blood pressure circadian rhythms," Experimental Diabetes Research, vol. 2012, Article ID 459087, 7 pages, 2012.

[89] J. M. Richey, M. Ader, D. Moore, and R. N. Bergman, "Angiotensin II induces insulin resistance independent of changes in interstitial insulin," American Journal of Physiology, vol. 277, no. 5, part 1, pp. E920-E926, 1999.

[90] J. F. Giani, M. C. Muñoz, M. A. Mayer et al., "Angiotensin-(17) improves cardiac remodeling and inhibits growth-promoting pathways in the heart of fructose-fed rats," American Journal of Physiology, vol. 298, no. 3, pp. H1003-H1013, 2010. 
[91] K. Shinozaki, K. Ayajiki, Y. Nishio, T. Sugaya, A. Kashiwagi, and T. Okamura, "Evidence for a causal role of the reninangiotensin system in vascular dysfunction associated with insulin resistance," Hypertension, vol. 43, no. 2, pp. 255-262, 2004.

[92] M. Paul, A. Poyan Mehr, and R. Kreutz, "Physiology of local renin-angiotensin systems," Physiological Reviews, vol. 86, no. 3, pp. 747-803, 2006.

[93] S. S. Xing, X. P. Bi, H. W. Tan, Y. Zhang, Q. C. Xing, and W. Zhang, "Overexpression of interleukin-18 aggravates cardiac fibrosis and diastolic dysfunction in fructose-fed rats," Molecular Medicine, vol. 16, no. 11-12, pp. 465-470, 2010.

[94] L. Tappy and K. A. Le, "Metabolic effects of fructose and the worldwide increase in obesity," Physiological Reviews, vol. 90, no. 1, pp. 23-46, 2010.

[95] Y. B. Lombardo, S. Drago, A. Chicco et al., "Long-term administration of a sucrose-rich diet to normal rats: relationship between metabolic and hormonal profiles and morphological changes in the endocrine pancreas," Metabolism, vol. 45, no. 12, pp. 1527-1532, 1996.

[96] Z. Vasanji, E. J. F. Cantor, D. Juric, M. Moyen, and T. Netticadan, "Alterations in cardiac contractile performance and sarcoplasmic reticulum function in sucrose-fed rats is associated with insulin resistance," American Journal of Physiology, vol. 291, no. 4, pp. C772-C780, 2006.

[97] R. D. Bunag, T. Tomita, and S. Sasaki, "Chronic sucrose ingestion induces mild hypertension and tachycardia in rats," Hypertension, vol. 5, no. 2, pp. 218-225, 1983.

[98] R. Buettner, J. Schölmerich, and L. C. Bollheimer, "High-fat diets: modeling the metabolic disorders of human obesity in rodents," Obesity, vol. 15, no. 4, pp. 798-808, 2007.

[99] S. C. Woods, R. J. Seeley, P. A. Rushing, D. D'Alessio, and P. Tso, "A controlled high-fat diet induces an obese syndrome in rats," Journal of Nutrition, vol. 133, no. 4, pp. 1081-1087, 2003.

[100] L. J. Prior, N. Eikelis, J. A. Armitage et al., "Exposure to a high-fat diet alters leptin sensitivity and elevates renal sympathetic nerve activity and arterial pressure in rabbits," Hypertension, vol. 55, no. 4, pp. 862-868, 2010.

[101] N. M. Fardin, L. M. Oyama, and R. R. Campos, "Changes in baroreflex control of renal sympathetic nerve activity in highfat-fed rats as a predictor of hypertension," Obesity, vol. 20, no. 8, pp. 1591-1597, 2012.

[102] M. Damjanovic and M. Barton, "Fat intake and cardiovascular response," Current Hypertension Reports, vol. 10, no. 1, pp. 25-31, 2008.

[103] C. M. Boustany, K. Bharadwaj, A. Daugherty, D. R. Brown, D. C. Randall, and L. A. Cassis, "Activation of the systemic and adipose renin-angiotensin system in rats with diet-induced obesity and hypertension," American Journal of Physiology, vol. 287, no. 4, pp. R943-R949, 2004.

[104] B. P. Sampey, A. M. Vanhoose, H. M. Winfield et al., "Cafeteria diet is a robust model of human metabolic syndrome with liver and adipose inflammation: comparison to high-fat diet," Obesity, vol. 19, no. 6, pp. 1109-1117, 2011.

[105] A. Sclafani and D. Springer, "Dietary obesity in adult rats: similarities to hypothalamic and human obesity syndromes," Physiology and Behavior, vol. 17, no. 3, pp. 461-471, 1976.

[106] A. Heyne, C. Kiesselbach, I. Sahún et al., "An animal model of compulsive food-taking behaviour," Addiction Biology, vol. 14, no. 4, pp. 373-383, 2009.
[107] M. J. Morris, H. Chen, R. Watts, A. Shulkes, and D. CameronSmith, "Brain neuropeptide Y and CCK and peripheral adipokine receptors: temporal response in obesity induced by palatable diet," International Journal of Obesity, vol. 32, no. 2, pp. 249-258, 2008.

[108] M. S. Muntzel, O. A. Al-Naimi, A. Barclay, and D. Ajasin, "Cafeteria diet increases fat mass and chronically elevates lumbar sympathetic nerve activity in rats," Hypertension, vol. 60, no. 6, pp. 1498-1502, 2012.

[109] R. E. Haddock, T. H. Grayson, M. J. Morris, L. Howitt, P. S. Chadha, and S. L. Sandow, "Diet-induced obesity impairs endothelium-derived hyperpolarization via altered potassium channel signaling mechanisms," PLoS ONE, vol. 6, no. 1, Article ID e16423, 2011.

[110] A. Miesel, H. Muller, M. Thermann, M. Heidbreder, P. Dominiak, and W. Raasch, "Overfeeding-induced obesity in spontaneously hypertensive rats: an animal model of the human metabolic syndrome," Annals of Nutrition and Metabolism, vol. 56, no. 2, pp. 127-142, 2010.

[111] A. Daffonchio, C. Franzelli, M. Di Rienzo, P. Castiglioni, G. Mancia, and A. U. Ferrari, "Sympathetic, parasympathetic and non-autonomic contributions to cardiovascular spectral powers in unanesthetized spontaneously hypertensive rats," Journal of Hypertension, vol. 13, no. 12, part 2, pp. 1636-1642, 1995.

[112] K. Ju and T. Kubo, "Power spectral analysis of autonomic nervous activity in spontaneously hypertensive rats," Biomedical Sciences Instrumentation, vol. 33, no. 1, pp. 338-343, 1997. 


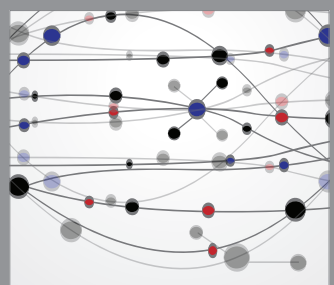

The Scientific World Journal
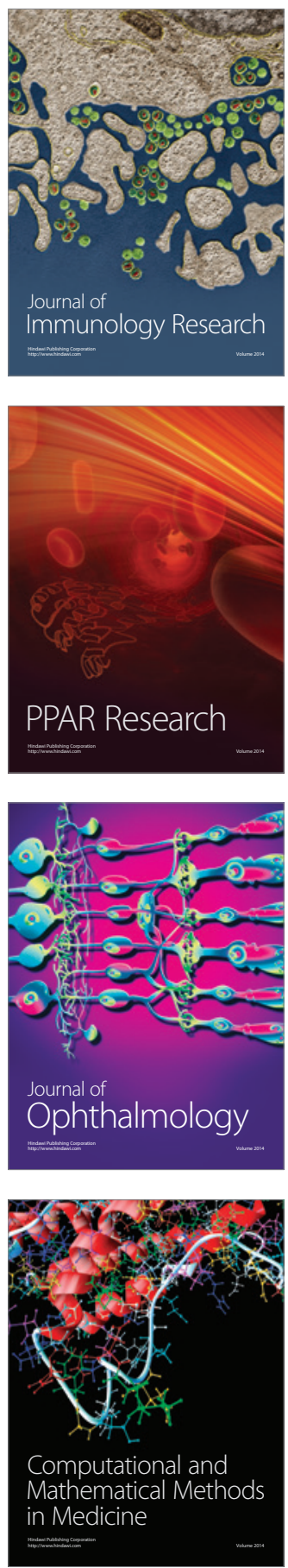

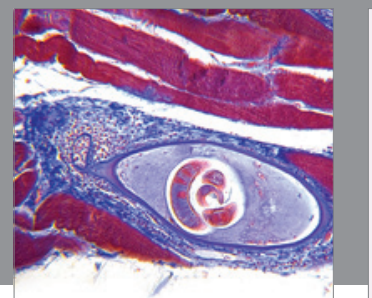

Gastroenterology

Research and Practice
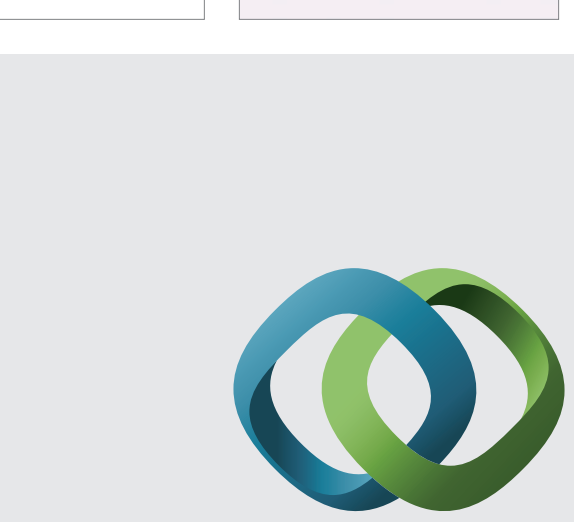

\section{Hindawi}

Submit your manuscripts at

http://www.hindawi.com
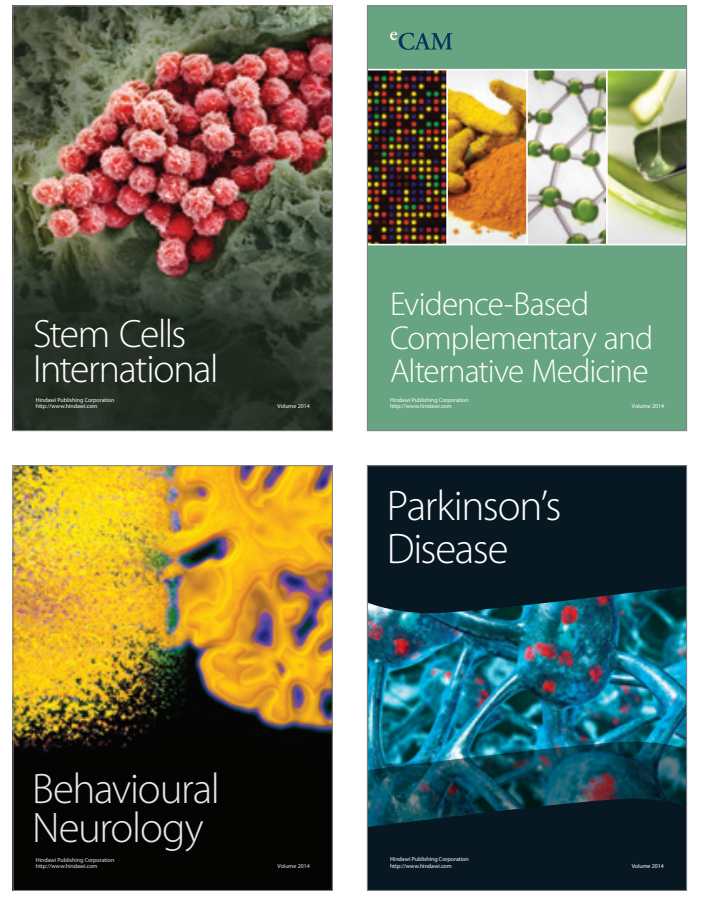
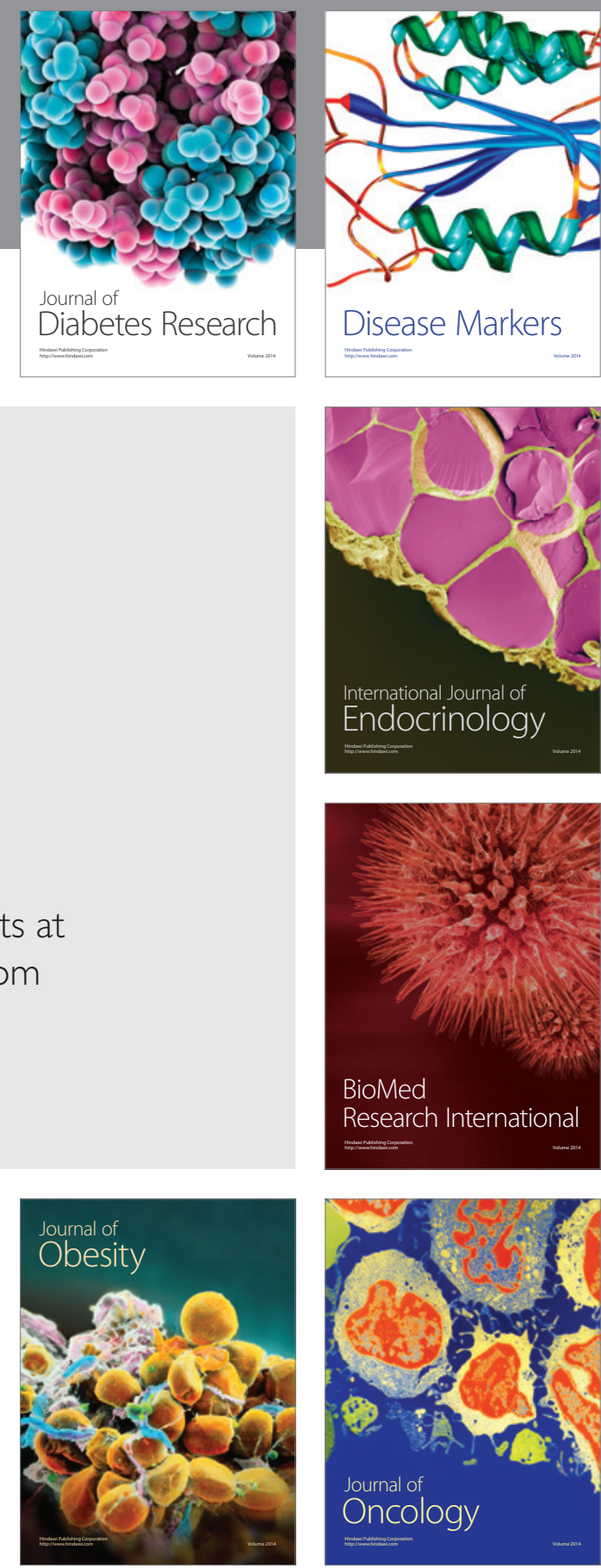

Disease Markers
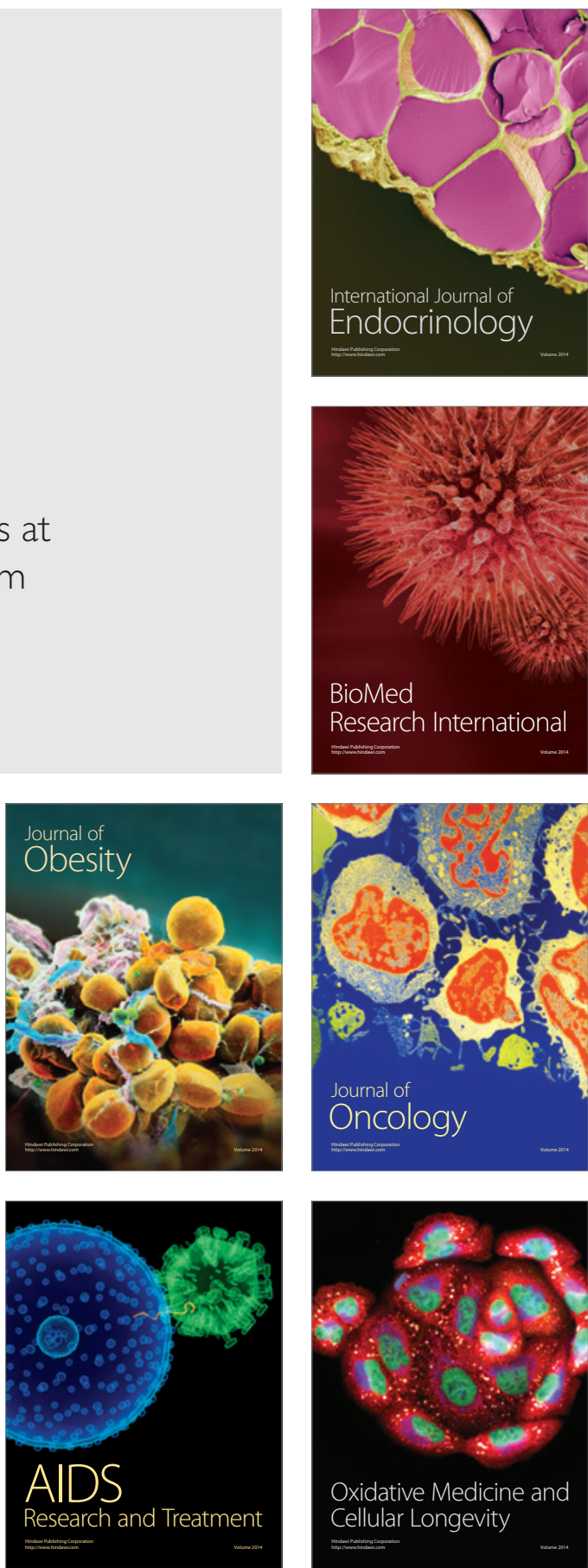